\title{
Comparación de differentes técnicas de cepillado para la higiene bucal
}

Comparison of different tooth brushing techniques for oral hygiene Lina María Rizzo-Rubio ${ }^{1 凶}$, Ana María Torres-Cadavid ${ }^{1 凶}$, Cecilia María Martínez-Delgado ${ }^{2 \otimes} \underline{\text { CvLAC }}$

1. Odontólogas Universidad CES, Medellín, Colombia.

2. Odontóloga Epidemióloga, Magister en Dirección Universitaria. Universidad CES, Medellín, Colombia.

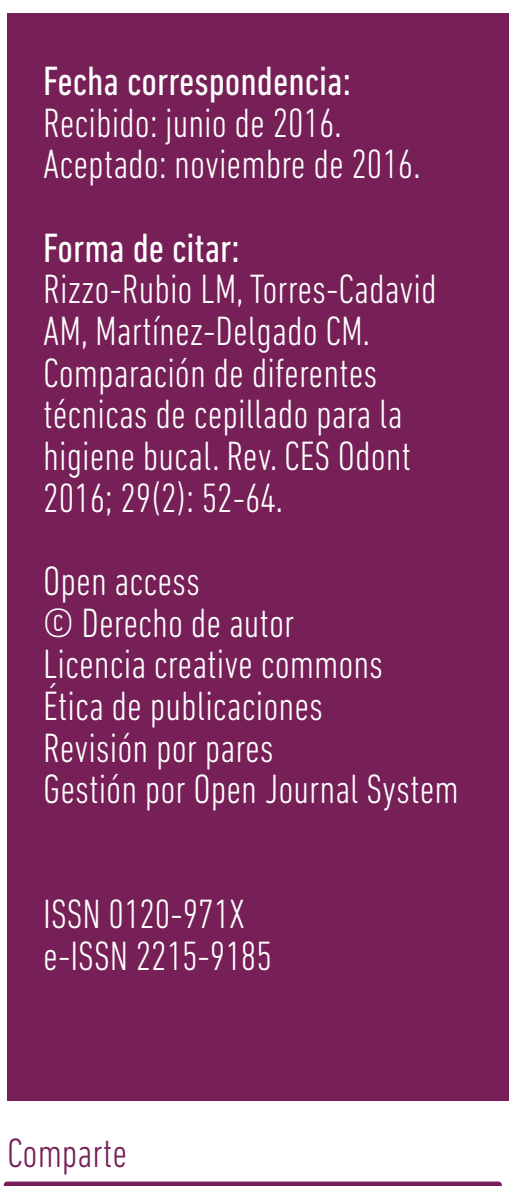

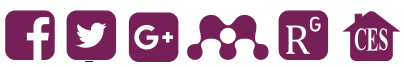

\section{Resumen}

El principal factor etiológico a controlar por parte de los profesionales de la salud bucal, es la placa bacteriana, la cual desencadena diferentes enfermedades como la caries dental, la enfermedad periodontal y otras enfermedades de carácter infeccioso.

Se ha observado que para la remoción de la placa bacteriana, el método más efectivo es el cepillado, el cual, realizándolo de manera adecuada garantiza la higiene oral; sumado a este método se encuentra el uso de enjuagues bucales, la seda dental y los dentífricos como complemento para el mantenimiento de la salud bucal.

A través del tiempo, diferentes autores se han preocupado por describir técnicas de cepillado, las más mencionadas son: La técnica de Bass modificada, la técnica vibratoria de Charters, la técnica de Stillman modificada, la técnica Fones y la técnica Scrub; se ha hecho énfasis en que lo importante es realizar un cepillado minucioso que garantice la remoción de la placa de forma adecuada: pero, si es necesario escoger una en especial, se hará según la situación clínica de cada paciente.

Para lograr los objetivos de la higiene oral no basta con describir las técnicas y métodos existentes para lograrla, si no, que es fundamental la educación y promoción de la salud oral, por lo que los odontólogos e higienistas bucales deben orientarse a lograr que las personas se concienticen de la necesidad del autocuidado y garantizar que realicen una técnica de cepillado que remueva efectivamente los residuos alimenticios y microorganismos que ayudan en la formación de la biopelícula.

Palabras clave: Cepillado dental, Salud bucal, Dispositivos dentales, Cuidado en el hogar, Cuidado primario.

\section{Abstract}

The main etiological factor to control by oral health professionals has been the bacterial plaque, which cause different illness such as dental caries, periodontal disease and another infectious.

It has been observed that for the removal of bacterial plaque, the most effective method has been tooth brushing, this one guarantees oral hygiene if you do it in a correct way, in addition to this there are more methods as mouthwash, dental floss and tooth paste as a complement to the maintenance of oral health. 
Trough time, different authors has been concerned about describing tooth brushing techniques, the most popular are: the bass modified technique, the charters vibratory technique, the stillman modified technique, the fones technique and the scrub technique; it has been emphasized that the most important thing it's the performance of a meticulous tooth brushing that guarantees the complete removal of plaque on an correct way: but the important thing is to choose one technique, this one will be done according to the clinical situation.

To achieve the objectives to a correct oral hygiene its not enough to describe the techniques ant different methods to do this, the fundamental thing is the education and the oral health promotion, that's why the dentists and oral hygienists must orient to achieve that people get aware of the need for self-care and guarantee that they perform a correct tooth brushing technique, capable of remove food waste and microorganisms that help the formation of biofilm.

Keywords: Tooth brushing, oral health, dental devices, home care, primary care.

\section{Introducción}

La placa bacteriana o biopelícula dental se considera el principal factor etiológico de las enfermedades infecciosas de la cavidad bucal (1); su presencia, en niveles y en grados de maduración, ayuda al establecimiento de diferentes patologías bucales como la caries dental, la gingivitis y la enfermedad periodontal (2, 3 ). Los odontólogos y especialistas de la salud bucal recomiendan diversas estrategias para su control, con resultados distintos dependiendo del acceso a la educación, a los servicios de salud bucal y a la compra de elementos de higiene bucal, a los ingresos, al tipo de aseguramiento, en fin a multiplicidad de variables personales y socioeconómicas (4).

Entre los diferentes métodos para la higiene bucal se recomienda el cepillado, el uso de la seda, y, actualmente, toma fuerza el concepto de la importancia de los dentífricos, entendiendo que el esmalte es un tejido vivo dinámico, que puede incorporar minerales provenientes del fluido bucal $(\underline{5}, \underline{6})$.

Existen diversas técnicas de cepillado dental así como diferentes cepillos, tantos como las casas comerciales pueden fabricar. Una de ellas es la técnica modificada de Bass (ㅁ) , creada por el Dr. Charles Cassidy Bass en 1950, porque consideraba que existía poco conocimiento para la prevención de las enfermedades de la cavidad bucal. Inicialmente tuvo rechazo entre la comunidad académica pero con el tiempo se observó que sus enseñanzas fueron ampliamente aceptadas por la profesión odontológica y siguen en uso hoy en día (7) debido a que esta técnica es la recomendada para pacientes que se encuentran en estado de salud periodontal (8).

La técnica Bass modificada es elegida por varios odontólogos como la técnica ideal para enseñar a los pacientes como realizar su cepillado, probando su validez y aplicación para la remoción de placa bacteriana de manera adecuada (9), inclusive en pacientes usuarios de aparatología ortodóntica (10). Así como esta técnica tiene adeptos, hay otras investigaciones que demuestran poca efectividad y escasa superioridad, por ejemplo los estudios de Morita (1998), referidos a la poca efectividad en áreas interproximales, enfrentándola con el uso del palillo dental (11). Este utensilio elaborado con madera permite realizar la limpieza en las áreas interproximales, sin embargo no se recomienda su uso por el peligro de dañar la estructura de la papila dental (12). 
Teniendo en cuenta la trayectoria de la técnica de Bass, su amplia aceptación y eficiencia, se pretende compararla con otras técnicas de cepillado, con el fin de establecer recomendaciones clínicas que propicien el uso de cada una de ellas según la necesidad en los diferentes públicos de tal manera que se logre una excelente higiene bucal.

\section{Metodología}

\section{Estrategia de búsqueda}

Con el fin de lograr los objetivos de la revisión de tema se realizó una búsqueda de literatura según los criterios de la figura 1, la cual arrojó los siguientes resultados al usar las palabras clave de manera individual y combinándolas entre ellas: Salud oral: (15.727 referencias), cepillado dental (481 referencias), dispositivos dentales (4.950 referencias), cuidado en el hogar ( 236 referencias) y cuidado primario (697 referencias); también, se utilizaron las siguientes palabras las cuales se encontraron en diferentes títulos de referencias y fueron utilizadas como complemento en la búsqueda: Técnica modificada de Bass, efectividad de las técnicas de cepillado, comparación entre las técnicas de cepillado, higiene oral y remoción de placa bacteriana. Al revisar los resúmenes y títulos de las referencias arrojadas se encontró que había varias que no tenían relevancia para el tema y se incluyeron 50 artículos de manera definitiva.

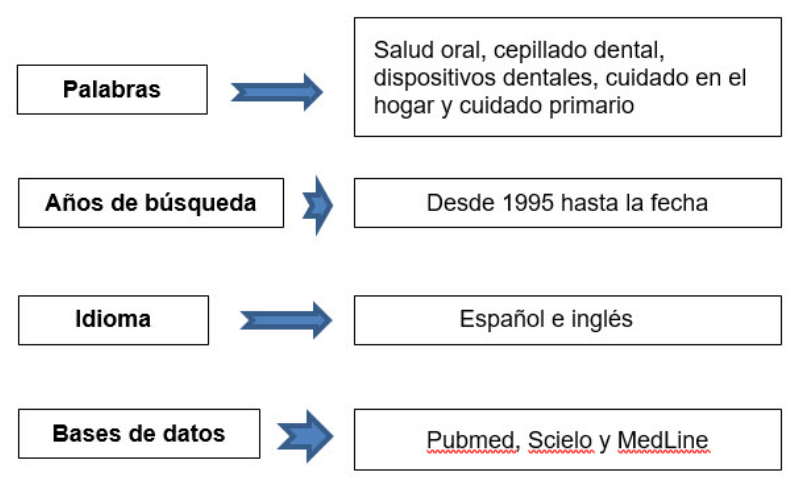

Figura 1. Estrategia de búsqueda bibliográfica

\section{Desarrollo del tema}

La higiene bucal es uno de los métodos del autocuidado; se educa principalmente a la población sobre el uso del cepillo, de la crema dental y de la seda, como elementos indispensables para el control de patologías infecciosas, tratando de que estas no se establezcan en la cavidad bucal $(12,13)$. La mayoría de los niños no reciben una revisión temprana de tipo dental mientras van a sus chequeos de rutina con el médico, existe falta de conocimiento por las enfermeras y profesionales de la salud que no pertenecen al área odontológica acerca del cuidado primario que se debe establecer en estas edades tempranas (14).

La caries dental es la enfermedad crónica más común que afecta a los niños de todo el mundo $(14,15)$, su etiología multifactorial ataca al tejido duro del diente: estructuras anatómicas conocidas como esmalte, dentina y cemento (16). Esta enfermedad es causada principalmente por bacterias que fermentan los carbohidratos procedentes de la dieta produciendo cambios en el pH salivar de tal manera que se genera una pérdida de minerales $(\underline{17}, \underline{18})$. 
En etapas tempranas, esta pérdida de minerales aun no produce una cavidad, a esto se conoce como caries incipiente del esmalte o mancha blanca. (19) En este estado la pérdida de minerales es reversible mediante un proceso de remineralización (16).

Existen otras enfermedades de la cavidad bucal relacionadas con la higiene bucal como la enfermedad periodontal $(\underline{20}, 21)$; se conoce como periodontitis y es una enfermedad inflamatoria de los tejidos gingivales y periodontales, en parte como respuesta a la película de la placa bacteriana (22). Existen dos tipos de periodontitis, una es crónica, en la que la destrucción de los tejidos ocurre a través de un largo periodo de tiempo y la otra es la agresiva, que resulta en una destrucción más rápida en la que hay pérdida ósea; en las dos, hay presencia de bacterias específicas que atacan los tejidos (23). Anteriormente esta enfermedad era relacionada de manera equivoca con la edad de las personas, pero ahora se ha demostrado que con una adecuada higiene oral se conserva la salud bucal (24).

Las enfermedades bucales actualmente se identifican con enfermedades del desarrollo por la presencia de carbohidratos complejos que hacen parte de la dieta, así como el cambio de costumbres y comportamientos, que, sumados a la falta de higiene oral, ha aumentado el interés y la preocupación por el control de la placa bacteriana $(\underline{20}, 21)$.

El método más común y más utilizado para la higiene bucal y la prevención de las enfermedades descritas anteriormente, es el cepillado dental (23). Se reconoce que es lo más útil para el control de la placa supragingival (25). A través del tiempo se han descrito diferentes técnicas de cepillado, las cuales difieren entre sí, dependiendo de la edad, de las habilidades y del estado de salud bucal del paciente, algunas son más recomendadas y reconocidas que otras (26); la técnica de cepillado indicada para personas en estado de salud periodontal, es la modificada de Bass, la cual ha ofrecido buenos resultados $(\underline{27}, \underline{28})$.

Para la técnica de Bass se recomienda un cepillo de cerdas suaves para evitar, primero, la abrasión de la estructura dental dura, y segundo, la lesión de la encía marginal por trauma (29). La técnica consiste en que el cepillo se coloca en un ángulo de 45 grados (Figura 2a) con respecto al eje longitudinal del diente (teniendo en cuenta que las cerdas van hacia la parte apical del diente); los filamentos del cepillo se introducen en los nichos interdentales y el surco gingival, al estar ahí se realizan pequeños movimientos vibratorios y después un movimiento de barrido hacia oclusal (Figura 2b). Con esta técnica está limitada la limpieza de las superficies oclusales (30).
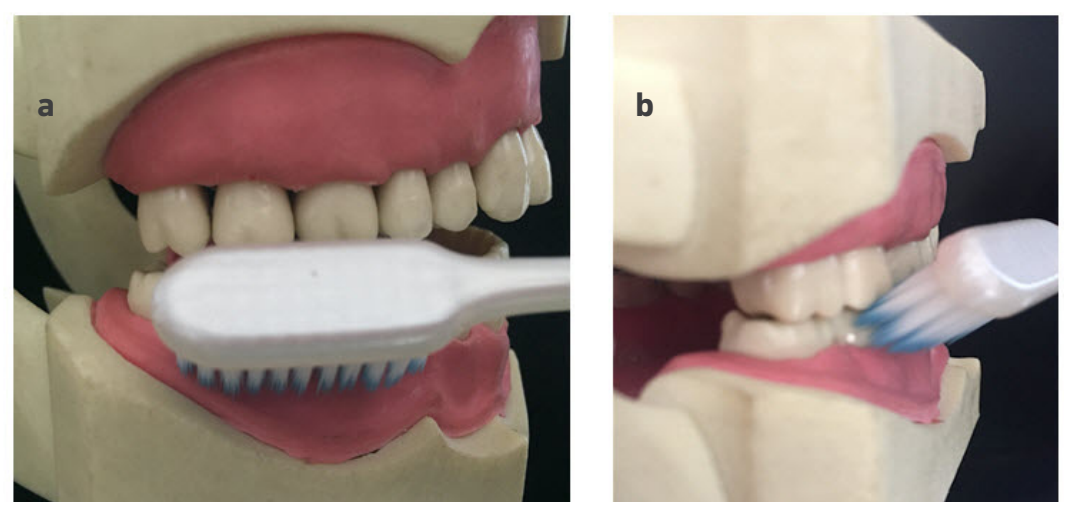

Q Figura 2a, 2b. Técnica de Bass. Fuente: Elaboración propia 
La segunda técnica más recomendada es la técnica de Fones (27), que está indicada para las superficies vestibulares; para llevarla a cabo, los dientes deben estar en oclusión o en posición de reposo, y los filamentos del cepillo se colocan formando un ángulo de 90 grados con respecto a la superficie bucal del diente (Figura 3). Estas superficies se dividen en 6 sectores y se realizan 10 amplios movimientos rotatorios en cada sector. En las caras oclusales, se realizan movimientos circulares y en las caras linguo - palatinas se coloca el cepillo en posición vertical y se realizan movimientos rotatorios; está indicada en niños $(\underline{30}, \underline{31})$ por la facilidad para aprenderla, en comparación con la técnica de Bass (32).

La tercera técnica es la técnica horizontal de Scrub (27), ésta consiste en que los filamentos del cepillo se colocan en un ángulo de 90 grados sobre la superficie vestibular, linguo- palatina y oclusal de los dientes (Figura 4). Se realiza una serie de movimientos repetidos de atrás para adelante sobre toda la arcada, la cavidad oral se divide en sextantes y se realizan 20 movimientos por cada sextante (30); se ha demostrado que es el método de elección en niños en edad preescolar, porque ellos tienen menor habilidad para llevar a cabo otros métodos de cepillado y se encuentran en la edad en la que están desarrollando sus capacidades motoras (28), pero a la vez, se ha observado que las técnicas de cepillado horizontal aumentan la abrasión del esmalte (33).

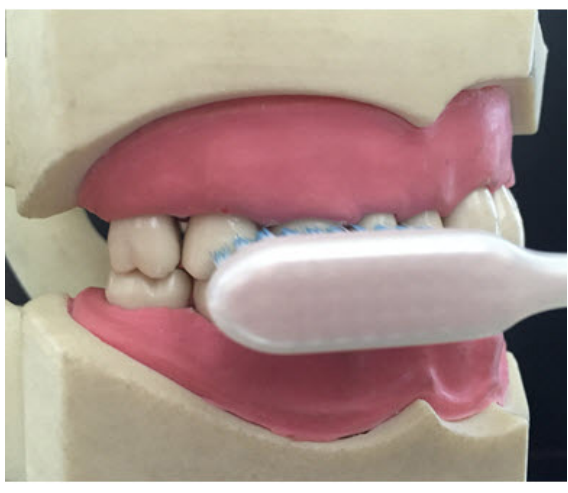

Q

Figura 3. Técnica de Fones. Fuente: Elaboración propia.

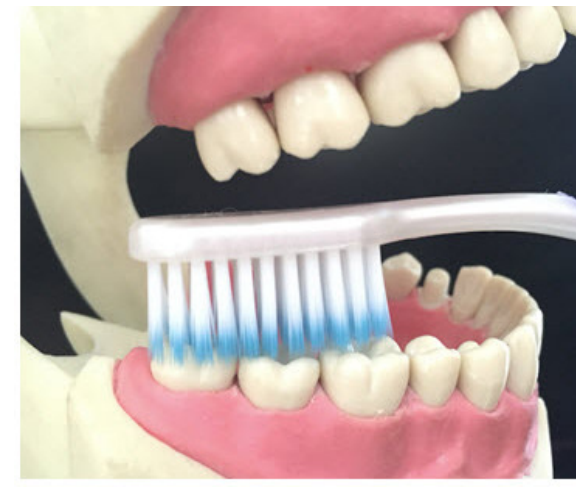

Figura 4. Técnica horizontal de Scrub. Fuente: Elaboración propia.

La técnica de cepillado de Stillman modificada (Figuras $5 a$ y 5 b) está indicada en pacientes adultos que no tienen enfermedad periodontal, es igual a la técnica de Bass pero los filamentos se colocan $2 \mathrm{~mm}$ por encima del margen gingival, es decir, encima de la encía adherida. Se realiza a presión hasta observar la palidez de los márgenes gingivales, la vibración se mantiene por 15 segundos por cada dos dientes y al finalizarla se realiza movimiento hacia oclusal de barrido $(\underline{30}, \underline{35})$.

La técnica vibratoria de Charters es la menos recomendada (27), fue descrita por Charters en 1928 y está indicada en pacientes adultos con enfermedades periodontales; el objetivo de esta técnica es la eliminación de la placa interproximal (Figuras 6a y 6b). Para realizarla, se debe ubicar el cepillo formando un ángulo de 45 grados con respecto al eje dental pero dirigido hacia el borde incisal, y se presiona ligeramente para que los filamentos penetren en el espacio interdental. Se realizan movimientos vibratorios que producen un masaje en las encías (30). 


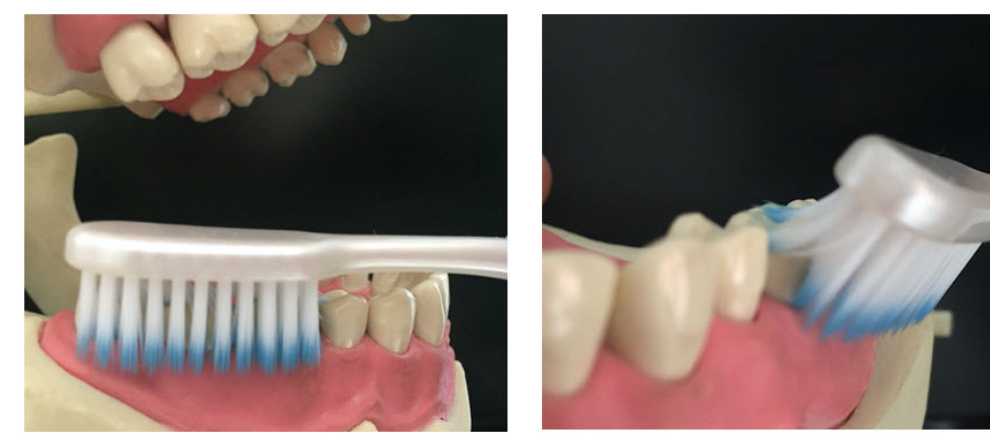

Q Figuras 5a, 5b. Técnica de Stillman modificada. Fuente: Elaboración propia.
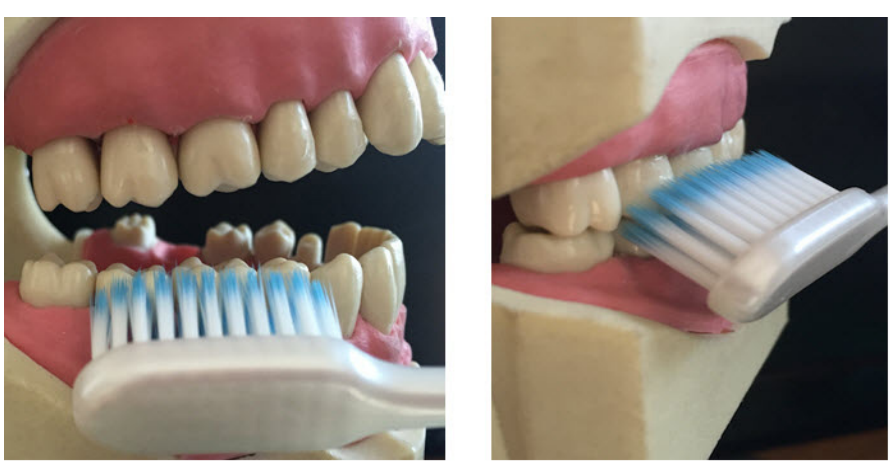

(9)

Figuras 6a, 6b. Técnica vibratoria de Charters. Fuente: Elaboración propia.

La descripción anterior acerca de las técnicas de cepillado indica que hay una para la situación clínica que presente cada paciente, pero más que determinar cuál es la que ofrece mejores resultados, lo importante es realizarla de manera adecuada y minuciosa; ya si hay un caso especial que necesite el empleo de una, se debe enseñar al paciente de qué forma realizarla ( $\underline{36})$.

Además del cepillado como método principal utilizado para obtener buenos resultados al realizar la higiene bucal (37), hay otros factores que influyen en ella como los complementos utilizados para realizarla, como la seda dental, la pasta dentífrica y enjuagues bucales. Estos últimos, se han desarrollado para prevenir la colonización y establecimiento de microorganismos denominados cariogénicos, como Streptococus mutans en la cavidad bucal disminuyendo la actividad de la caries y los recuentos microbianos (38); la pasta dentífrica, junto con el cepillo, hacen parte de un importante hábito de higiene bucal que ofrece muchos beneficios para la salud dental y gingival (39) como lo es la remineralización de la caries (40) y la prevención de la aparición de la gingivitis provocada por la placa dental (41).

Con el advenimiento de nuevas tecnologías llegaron al mercado cepillos dentales eléctricos los cuales han demostrado tener una alta energía y fuerzas de cizallamiento suficientes capaces de remover una significante cantidad de bacterias en la cavidad oral (42), como también han demostrado ser más eficientes que la técnica de Bass en cuanto a la remoción de placa, demostrando una mejoría en las técnicas utilizadas, pues para las personas que no presentan la capacidad ni la habilidad motora de realizar correctamente la técnica de cepillado, esto puede ser de gran utilidad (43). Además, el estudio de Grazyna Smiech y Joanna Jabloska (2007) demostró que estos cepillos eléctricos ofrecen tan buenos resultados como cuando se usa un cepillo tradicional junto con la seda dental (44). 
Con respecto a la periodicidad del cepillado dental, se recomienda al menos dos veces al día para lograr un control de placa de manera aceptable $(\underline{43}, \underline{45})$ cuando el cepillado es excesivo y además se agrega a esto que el cepillo tenga cerdas duras, que la técnica de cepillado sea horizontal y la pasta dentrífica abrasiva, se puede presentar abrasión del tejido dental, recesión gingival y aumento de la sensibilidad (46). La exposición de la dentina en la zona cervical por medio del cepillado, hace más vulnerable el tejido dental frente a la pasta dentífrica y al pH ácido de los alimentos, debilitándolo y haciéndolo más susceptible a otras lesiones como la abfracción y la hipersensibilidad dentinal, que aunque su origen no esté relacionado directamente con el trauma causado por el cepillado, es un factor importante en la progresión de ellas (느).

Independientemente de la técnica del cepillado, se ha demostrado que una buena higiene oral comienza desde la educación en promoción de la salud, la adopción de buenos hábitos y la buena realización de la técnica que se emplee, la cual debe ser

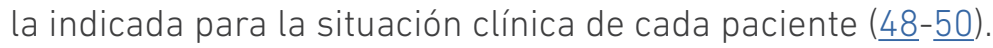

\section{Discusión}

Con base en las referencias revisadas, se encuentra que a través de los años se ha luchado por conseguir la mejor manera de evitar la maduración de la placa bacteriana dental, la cual se adhiere a la superficie dental ocasionando daños a las estructuras de los tejidos duros y blandos. Se ha comprobado que el método más efectivo para mantener la salud bucal es una adecuada técnica de cepillado, con múltiples métodos para realizarla, basados en diferentes aspectos como la edad (personas adultas o niños), el estado de salud de las encías, el establecimiento de la enfermedad periodontal, los tratamientos quirúrgicos, la facilidad de aprender la técnica por parte de los pacientes y enseñarla por parte del odontólogo.

Existe una gran discusión acerca de la efectividad de cada una de estas técnicas, tomando en cuenta las ventajas y desventajas de cada una, al igual que la forma como se deben realizar, pero independiente de eso, se sigue demostrando por medio de las referencias revisadas que la técnica de Bass es la más acogida por profesionales de la salud oral porque con ella se obtiene mayor efectividad en la remoción de la placa bacteriana, siempre y cuando se haga adecuadamente.

La técnica modificada de Bass presenta importantes ventajas como la protección de los tejidos gingivales al tener una angulación específica y una dirección hacia la superficie dental y no hacia la encía, en comparación con la técnica de Stillman, cuyo método incluye un cepillado sobre el margen gingival el cual podría llevar a recesiones gingivales; abarca superficies interproximales al tener una angulación adecuada que permite la entrada de las cerdas en estos espacios, mientras que la técnica de Scrub consiste en realizar movimientos horizontales donde no se podría entrar por completo desde el inicio del espacio interproximal que está dirigido hacia la papila ó con la técnica de Charters, la cual se dirige en sentido opuesto a la técnica de Bass: su trayectoria comienza en el borde incisal hacia el margen gingival lo cual podría ocasionar daño en la encía, la técnica de Bass realiza una limpieza completa de la corona clínica al realizarse con movimientos de barrido hacia la superficie oclusal, lo cual no ocurriría con la técnica de fones, cuyo movimiento es rotatorio, el cual podría omitir zonas de los dientes por su trayectoria.

La técnica de Bass también posee desventajas, una de ellas es su difícil aprendizaje, ya que requiere esfuerzo, dedicación y realización cautelosa de la misma para lograr 
su efectividad, comparada con la técnica de Fones, la cual hay investigaciones que demuestran mayor rapidez al aprendizaje que con la técnica de Bass.

Otra desventaja de esta técnica se referencia en el estudio realizado por Morita (10) que considera a la técnica de Bass como la menos efectiva en la remoción de placa bacteriana interproximal comparada con la técnica del palillo dental. Esta comparación no le quita a la técnica de Bass su superioridad, ya que tiene como referente, espacios interproximales amplios y necesita especial atención para no lastimar la papila interdental, pues al introducir palillos de madera entre los dientes puede producir sangrado y lesión de las encías por la rigidez y punta del elemento. Se sugiere que la técnica de Bass debe usarse con cepillos de cerdas suaves, con objetivo de proteger las papilas interdentales y la encía marginal evitando de esta forma la abrasión dental y el daño de tejidos gingivales.

Adicional a esto, la técnica modificada de Bass además de tener una angulación adecuada para llegar correctamente los espacios interproximales propone el uso de seda dental como complemento para un óptimo resultado, siendo esta necesaria para llegar a las zonas a las que el cepillo de dientes no puede alcanzar, y se ha descrito su necesidad e importancia.

También se ha comprobado con el advenimiento de nuevas tecnologías como son los cepillos eléctricos su superioridad en comparación con cepillado manual, pero también se comprueba que al usarlos con la técnica de Bass son más efectivos.

Es importante discutir que aunque se emplee una técnica de cepillado óptima, es necesario realizarla como mínimo 2 veces al día para lograr un alto nivel de higiene oral, como también no se debe realizar el cepillado dental en exceso ya que este podría causar abrasión de tejido dental, recesión gingival y aumento en la sensibilidad.

\section{Conclusiones}

Cada autor defiende su técnica con argumentos diferentes, pero al final la importancia de designar cual es la mejor, tiene que ver con la efectividad de la remoción de placa bacteriana y en la correcta realización de la técnica que se desee, siguiendo las instrucciones e indicaciones descritas para cada una.

Independiente del avance tecnológico de los últimos años, el cepillado dental sigue siendo el método de elección para mantener la higiene oral; aunque existen diferentes técnicas de cepillado descritas y la técnica de Bass sea la más recomendada y utilizada, lo importante es realizar el cepillado de manera minuciosa de una forma que garantice la remoción de la placa bacteriana de manera correcta, teniendo acceso a todas las superficies dentales y periodontales.

La base de lo anterior es la educación en la prevención de la enfermedad y promoción de la salud bucal, lo cual permite concientizar a las personas de manera que se evite el desarrollo de todas las enfermedades que se pueden desencadenar por falta de higiene.

Declaración sobre conflicto de intereses: Los autores no reportan conflictos a declarar, relacionados con el presente artículo. 


\section{Referencias bibliográficas}

1. Sbordone L, Bortolaia C. Oral microbial biofilms and plaque-related diseases: microbial communities and their role in the shift from oral health to disease. Clin Oral Investig. 2003 Dec;7(4):181-188. https://www.ncbi.nlm.nih.gov/pubmed/14598129

2. Petersen PE, Bourgeois D, Ogawa H, Estupinan-Day S, Ndiaye C. The global burden of oral diseases and risks to oral health. Bull World Health Organ. 2005 Sep;83(9):661-669. https://www.ncbi.nlm.nih.gov/pubmed/16211157

3. Chidiak F, Dugarte J, Márquez A, Murzi M, Osuna G, Vielma P. Salud bucal según el conocimiento y aplicación de técnicas de higiene oral. Creando. 2008;173-181. http://erevistas.saber.ula.ve/index.php/creando/article/view/1679

4. Sanabria-Castellanos CM, Suárez-Robles MA, Estrada-Montoya JH. Relación entre determinantes socioeconómicos, cobertura en salud y caries dental en veinte países. Rev Gerenc Polít Salud. 2015;14 (28):161-189. http://www.scielo.org.co/ $\mathrm{pdf} / \mathrm{rgps} / \mathrm{v} 14 \mathrm{n} 28 / \mathrm{v} 14 \mathrm{n} 28 \mathrm{a} 12 . \mathrm{pdf}$

5. Gluckstein Caracushansky Linda, Martinez Pabon María Cecilia, Mesa Cadavid Liliana. Efecto del dentrifico colgate total sobre el crecimiento del streptococo mutans. Rev Ces Odontol. 2010;10 (2). http://revistas.ces.edu.co/index.php/ odontologia/article/viewFile/1140/718

6. Jo S-Y, Chong H-J, Lee E-H, Chang N-Y, Chae J-M, Cho J-H, et al. Effects of various toothpastes on remineralization of white spot lesions. Korean J Orthod. 2014 May;44(3):113-118. https://www.ncbi.nlm.nih.gov/pmc/articles/PMC4040358/

7. Watts TLP. Periodontitis for medical practitioners. BMJ. 1998 Mar 28:316(7136):993-996.

8. Newman MG, Takei H, Klokkevold PR, Carranza FA. Carranza`s Clinical Periodontology. 11th ed. Amolca; 2014. https://www.ncbi.nlm.nih.gov/pmc/articles/ PMC1112886/

9. Bejarano NMP, Reissner CVD, Gaona MIF, Vazquez DAS, Cespedes ANN, Leguizamon LMF, et al. Conocimiento, actitud y pràctica sobre medidas de odontologia preventiva en odontòlogos:VI Congreso Paraguayo de Endodoncia, 2011. Rev Salud Pública Parag. 2014 Sep 1;4(1):27-34. http://www.ins.gov.py/revistas/index. $\mathrm{php/rspp/article/view/297}$

10. Nassar PO, Bombardelli CG, Walker CS, Neves KV, Tonet K, Nishi RN, et al. Periodontal evaluation of different toothbrushing techniques in patients with fixed orthodontic appliances. Dent Press J Orthod. 2013;18(1):76-80. http://www.scielo. $\underline{\mathrm{br} / \mathrm{scielo} . \text { php?script=sci arttext\&pid=S2176-94512013000100017 }}$

11. Morita M, Nishi K, Watanabe T. Comparison of 2 toothbrushing methods for efficacy in supragingival plaque removal. The Toothpick method and the Bass method. J Clin Periodontol. 1998 Oct;25(10):829-831. https://www.ncbi.nlm.nih.gov/ pubmed/9797056 
12. Martínez FB. Programa de prevención y control de placa dentobacteriana en niños de 7 a 8 años de edad de la primaria "Alfonso Arroyo Flores de Poza Rica, Ver." [Internet] [Thesis]. 2011 [cited 2015 May 5]. Available from: http://cdigital. uv.mx/handle/123456789/30904

13. Folayan MO, Adeniyi AA, Chukwumah NM, Onyejaka N, Esan AO, Sofola O0, et al. Programme guidelines for promoting good oral health for children in Nigeria: a position paper. BMC Oral Health. 2014;14:128. http://bmcoralhealth.biomedcentral.com/articles/10.1186/1472-6831-14-128

14. Rabiei S, Mohebbi SZ, Yazdani R, Virtanen Jl. Primary care nurses' awarenesS of and willingness to perform children's oral health care. BMC Oral Health. 2014;14:26. https://www.ncbi.nlm.nih.gov/pmc/articles/PMC3986874/

15. Goel R, Vedi A, Veeresha K-L, Sogi G-M, Gambhir R-S. Oral hygiene practices and dental caries prevalence among 12 \& 15 years school children in Ambala, Haryana -A cross-sectional study. J Clin Exp Dent. 2015 Jul;7(3):e374-379.

16. Tascón JE, Cabrera GA. Algunas creencias sobre susceptibilidad y severidad de la caries en adolescentes del Valle del Cauca, Colombia. Colomb Médica. 2005;36(3):140-145. http://colombiamedica.univalle.edu.co/index.php/comedica/article/view/356/1125

17. Reynolds EC. Calcium phosphate-based remineralization systems: scientific evidence? Aust Dent J. 2008 Sep;53(3):268-273. https://www.ncbi.nlm.nih.gov/ pubmed/18782374

18. Zaghrisson BU, Zachrisson S. Caries incidence and oral hygiene during orthodontic treatment. Eur J Oral Sci. 1971 Aug 1;79(4):394-401. https://www.ncbi. nlm.nih.gov/pubmed/5288673

19. Kianoush N, Adler CJ, Nguyen K-AT, Browne GV, Simonian M, Hunter N. Bacterial Profile of Dentine Caries and the Impact of $\mathrm{pH}$ on Bacterial Population Diversity. PLoS ONE. 2014 Mar 27;9(3):e92940. http://journals.plos.org/plosone/article?id=10.1371/journal.pone. 0092940

20. Holly FJ, Gray JA. Mechanism for incipient carious lesion growth utilizing a physical model based on diffusion concepts. Arch Oral Biol. 1968 Mar 1;13(3):319-333. http://www.aobjournal.com/article/0003-9969(68)90130-1/abstract?cc=y=

21. Löe H. Oral hygiene in the prevention of caries and periodontal disease. Int Dent J. 2000 Jun;50(3):129-139. https://www.ncbi.nlm.nih.gov/pubmed/10967765

22. Bregy L, Müggler AR, Martinez-Lozano Sinues P, García-Gómez D, Suter Y, Belibasakis GN, et al. Differentiation of oral bacteria in in vitro cultures and human saliva by secondary electrospray ionization - mass spectrometry. Sci Rep. 2015:5:15163. http://www.nature.com/articles/srep15163

23. Maddi A, Frank A. Scannapieco. Oral biofilms, oral and periodontal infections, and systemic disease. Am J Dent. 2013;26 (5):249-254. https://www.ncbi.nlm.nih. gov/pubmed/24479275 
24. Adair PM, Pine CM, Burnside G, Nicoll AD, Gillett A, Anwar S, et al. Familial and cultural perceptions and beliefs of oral hygiene and dietary practices among ethnically and socio-economicall diverse groups. Community Dent Health. 2004 Mar;21(1 Suppl):102-111. https://www.ncbi.nlm.nih.gov/pubmed/15072479

25. Joybell C, Krishnan R, V SK. Comparison of Two Brushing Methods- Fone's vs Modified Bass Method in Visually Impaired Children Using the Audio Tactile Performance (ATP) Technique. J Clin Diagn Res JCDR. 2015 Mar;9(3):ZC19-22. https:// www.ncbi.nlm.nih.gov/pmc/articles/PMC4413148/

26. Alava Diaz N del C. Enfermedades bucales en pacientes hipertensos afiliados al seguro social campesino pertenecientes al dispensario del sitio "las mercedes $n^{\circ} 2$ " de la parroquia honorato vásquez del cantón santa ana, provincia de Manabí- Ecuador, periodo septiembre-diciembre del 2013. [Quito]: Universidad Central del Ecuador; 2014.

27. Petersen P. The World Oral Health Report 2003: continuous improvement of oral health in the 21 st century - the approach of the WHO Global Oral Health Programme. Comunity Dent Oral Epidemiol. 2003;31:3-24. https://www.ncbi.nlm. nih.gov/pubmed/15015736

28. Wainwright J, Sheiham A. An analysis of methods of toothbrushing recommended by dental associations, toothpaste and toothbrush companies and in dental texts. Br Dent J. 2014 Aug 8;217(3):E5-E5. https://www.ncbi.nlm.nih.gov/pubmed/25104719

29. Patil SP, Patil PB, Kashetty MV. Effectiveness of different tooth brushing techniques on the removal of dental plaque in 6-8 year old children of Gulbarga. J Int Soc Prev Community Dent. 2014;4(2):113-116. https://www.ncbi.nlm.nih.gov/ pubmed/25254196

30. Dyer D, Addy M, Newcombe RG. Studies in vitro of abrasion by different manual toothbrush heads and a standard toothpaste. J Clin Periodontol. 2000;27(2):99103. https://www.ncbi.nlm.nih.gov/pubmed/10703654

31. Barba González EL, Romo Rodríguez MG, Trujillo Contreras F, Rolón Díaz JC, Fernández Carranza JG, Fuentes Lerma MG. Estudio comparativo de la técnica de cepillado de Fones para mejorar la higiene bucal en escolares de 7 a 13 años de edad: XII encuentro, participación de la mujer en la ciencia. Guadalajara: Universidad de Guadalajara.2015.

32. Gil LF, Agulló M. A, Cañamás SM., Ibañez CP. Sistemática de la higiene bucodental: el cepillado dental manual [Internet]. SEPA - Sociedad Española de Periodoncia y Osteointegración. 2005 [cited 2015 May 5]. Available from: https://sepa. es/component/k2/item/84-sistemÃAtica-de-la-higiene-bucodental-el-cepillado-dental-manual.html

33. Bergström J, Lavstedt S. An epidemiologic approach to toothbrushing and dental abrasion. Conininnity Dent Oral Epidemiol. 1979;7(1):57-64. https://www.ncbi. nlm.nih.gov/pubmed/282958 
34. Harnacke D, Mitter S, Lehner M, Munzert J, Deinzer R. Improving Oral Hygiene Skills by Computer-Based Training: A Randomized Controlled Comparison of the Modified Bass and the Fones Techniques. PLoS ONE. 2012 May 21;7(5):e37072. http://journals.plos.org/plosone/article?id=10.1371/journal.pone. 0037072

35. Zambrano Loor CJ. "Bass modificada y Stillman modificada; Eficacia en reducción de placa bacteriana, escolares unidad educativa \#408, Guayaquil 2014." [Guayaquil, Ecuador]: Universidad Católica de Santiago de Guayaquil; 2014.

36. Miñana V, Grupo Prevlnfad. Promoción de la salud bucodental. Rev Pediatr Aten Primaria. 2011;8 (51):435-458.

37. Soria-Hernández MA, Molina-F NM, Rodríguez-P R. Hábitos de higiene bucal y su influencia sobre la frecuencia de caries dental. Acta Pediatr Mex. 2008;29 (1):2124. http://www.medigraphic.com/pdfs/actpedmex/apm-2008/apm081e.pdf

38. Jauhari D, Srivastava N, Rana V, Chandna P. Comparative Evaluation of the Effects of Fluoride Mouthrinse, Herbal Mouthrinse and Oil Pulling on the Caries Activity and Streptococcus mutans Count using Oratest and Dentocult SM Strip Mutans Kit. Int J Clin Pediatr Dent. 2015 Aug;8(2):114-118. https://www.ncbi.nlm.nih. gov/pmc/articles/PMC4562043/

39. De Souza-Rodrigues RD, Ferreira S da S, D'Almeida-Couto RS, Lachowski KM, Sobral MÂP, Marques MM. Choice of toothpaste for the elderly: an in vitro study. Braz Oral Res. 2015;29. https://www.ncbi.nlm.nih.gov/pubmed/26176359

40. Vanichvatana S, Auychai P. Efficacy of two calcium phosphate pastes on the remineralization of artificial caries: a randomized controlled double-blind in situ study. Int J Oral Sci. 2013 Dec;5(4):224-228. https://www.ncbi.nlm.nih.gov/pub$\underline{\mathrm{med} / 24030557}$

41. Morawiec T, Dziedzic A, Niedzielska I, Mertas A, Tanasiewicz M, Skaba D, et al. The biological activity of propolis-containing toothpaste on oral health environment in patients who underwent implant-supported prosthodontic rehabilitation. Evid-Based Complement Altern Med ECAM. 2013;2013:704947. https://www. hindawi.com/journals/ecam/2013/704947/

42. Hope CK, Petrie A, Wilson M. Efficacy of removal of sucrose-supplemented interproximal plaque by electric toothbrushes in an in vitro model. Appl Environ Microbiol. 2005 Feb;71(2):1114-1116. https://www.ncbi.nlm.nih.gov/pmc/articles/ PMC546661/

43. Jain Y. A comparison of the efficacy of powered and manual toothbrushes in controlling plaque and gingivitis: a clinical study. Clin Cosmet Investig Dent. 2013 Feb 27;5:3-9. https://www.ncbi.nlm.nih.gov/pubmed/23674927

44. Smiech-Slomkowska G, Jablonska-Zrobek J. The effect of oral health education on dental plaque development and the level of caries-related Streptococcus mutans and Lactobacillus spp. Eur J Orthod. 2007 Apr 1;29(2):157-160. https:// www.ncbi.nlm.nih.gov/pubmed/17488998 
45. Cunha-Cruz J, Milgrom P, Shirtcliff RM, Huebner CE, Ludwig S, Allen G, et al. "Everybody brush!": protocol for a parallel-group randomized controlled trial of a family-focused primary prevention program with distribution of oral hygiene products and education to increase frequency of toothbrushing. JMIR Res Protoc. 2015;4(2):e58. https://www.ncbi.nlm.nih.gov/pubmed/26002091

46. Bueno L. Efectos lesivos del control mecanico de la placa microbiana. Periodoncia, Osteointegración e Implantes. 2005;10 (20).

47. Van Loveren C. Exposed cervical dentin and dentin hypersensitivity summary of the discussion and recommendations. Clin Oral Investig. 2013 Mar;17 Suppl 1:S73-6. https://www.ncbi.nlm.nih.gov/pmc/articles/PMC3585836/

48. Martin A. Tooth brushing, tooth wear and dentine hypersensitivity - are they associated? Int Dent J. 2005;55 (S4):261-267. https://www.ncbi.nlm.nih.gov/pubmed/16167604

49. Goche KR, Alvarado BS. Aplicación de un programa educativo en salud oral en adolescentes de una institución educativa peruana. Rev Estomatológica Hered. 2014 Jun 18;22(2):82. http://www.upch.edu.pe/vrinve/dugic/revistas/index. $\mathrm{php/REH/article/view/130}$

50. Contreras Adolfo. La promoción de la salud bucal como una estrategia para el desarrollo de la salud pública: una oportunidad para la profesión y para el país. Biomed- Rev Inst Nac Salud. 2013;33 (1):187-190. http://www.revistabiomedica. org/index.php/biomedica/article/view/1617 